\title{
A systematic review of arterial stiffness, wave reflection and air pollution
}

\author{
LUCA ZANOLI $^{1 *}$, PAOLO LENTINI ${ }^{2 *}$, ANTONIO GRANATA ${ }^{3}$, AGOSTINO GAUDIO $^{1}$, \\ PASQUALE FATUZZO $^{1}$, LEONARDO SERAFINO ${ }^{1}$, STEFANIA RASTELLI ${ }^{1}$, VALERIO FIORE ${ }^{1}$, \\ AMBRA D'ANCA ${ }^{1}$, SALVATORE SANTO SIGNORELLI ${ }^{1}$ and PIETRO CASTELLINO ${ }^{1}$ \\ ${ }^{1}$ Internal Medicine, Section of Nephrology, Department of Clinical and Experimental Medicine, \\ Policlinico Universitario, University of Catania, Catania; ${ }^{2}$ Nephrology and Dialysis Unit, San Bassiano Hospital, \\ Bassano del Grappa; ${ }^{3}$ Nephrology and Dialysis Unit, 'St. Giovanni di Dio’ Hospital, Agrigento, Italy
}

Received January 9, 2017; Accepted February 20, 2017

DOI: $10.3892 / \mathrm{mmr} .2017 .6392$

\begin{abstract}
Arterial stiffening is associated with increased cardiovascular risk. Whether exposure to relatively high levels of air pollution is associated with arterial stiffening is unclear. We aimed to assess the association between exposure to major air pollutants and arterial stiffening. PubMed, SCOPUS and Web of Science databases (through 31 January 2017) were searched using a combination of terms related to exposure to gaseous [nitrogen dioxide $\left(\mathrm{NO}_{2}\right)$, nitrogen oxides $\left(\mathrm{NO}_{\mathrm{x}}\right)$ and sulphur dioxide $\left.\left(\mathrm{SO}_{2}\right)\right]$ or particulate matter pollutants $\left(\mathrm{PM}_{2.5}\right.$, $\mathrm{PM}_{10}$ and $\mathrm{PM}_{10-2.5}$ ), arterial stiffness (pulse wave velocity) and reflected waves (augmentation index, augmentation pressure). Pertinent information were extracted from selected studies. In this systematic review were included 8 studies with available data on air pollution and arterial stiffness/reflected waves parameters (8 studies explored the effects of exposure to particulate matter pollutants, 3 studies the effects of exposure to gaseous pollutants); seven of them reported increased arterial stiffness/reflected waves after exposure to air pollution ( 6 of 8 studies after particulate matter pollutants; 2 of 3 studies after gaseous pollutants). Arterial stiffness and reflected waves were increased in the majority of the studies after both short- and long-term exposure to air pollutants. In conclusion, available evidence supports an association of main air pollutants with increased arterial stiffness and reflected waves. This finding may have implications for population-based strategies for the reduction of arterial stiffness, a vascular biomarker and an intermediate endpoint for cardiovascular disease.
\end{abstract}

Correspondence to: Dr Luca Zanoli, Internal Medicine, Section of Nephrology, Department of Clinical and Experimental Medicine, Policlinico Universitario, University of Catania, Via Santa Sofia 78, I-95123 Catania, Italy

E-mail: zanoli.rastelli@gmail.com

*Contributed equally

Key words: air pollution, arterial stiffness, augmentation index, inflammation, particulate matter, pulse wave velocity, systematic review

\section{Introduction}

Arterial stiffness is a vascular biomarker and an intermediate endpoint for cardiovascular disease (1). Similarly, pollution is an emerging cause of cardiovascular disease and mortality $(2,3)$. We have recently reported in patients with inflammatory bowel disease, a model of a concomitant chronic inflammation and low burden of traditional cardiovascular risk factors (4), that arterial stiffness and reflected waves are increased (5-7), dependent upon inflammation and reduced by immunomodulatory drugs (8) but not by salicylates (9). Despite some methodology issues (10), these results were confirmed by an independent group (11). Several possible pathways exist that could link inflammation and arterial stiffening (12). Because pollution is associated with elevated levels of serum biomarkers of inflammation $(13,14)$ and endothelial dysfunction (15-18), a condition associated with functional arterial stiffening (12), it is reasonable that inflammation could be a potential link between pollution and arterial stiffening and the consequent increase of the cardiovascular risk.

Pulse wave velocity (PWV) represents the speed at which the pressure wave generated by left-ventricular ejection is propagated within the arterial tree and is considered the gold standard for assessing regional arterial stiffness in clinical practice. Augmentation pressure and augmentation index are two measures of reflected pulse waves.

In this work, we aimed to perform a systematic review to investigate the role of air pollution on arterial stiffness and wave reflection.

\section{Materials and methods}

Review criteria. According to PRISMA guidelines, a systematic literature search of original studies in humans was performed using PubMed database (last accessed on January 31, 2017) without restrictions on the year of publication. The search terms were 'arterial stiffness' or 'pulse wave velocity' or 'augmentation index' or 'augmentation pressure' in combination with ' $\mathrm{PM}_{2.5}$ ' or ' $\mathrm{PM}_{10}$ ' or 'nitrogen dioxide' or 'nitrogen oxides' or 'sulfur dioxide' or ' $\mathrm{NO}_{2}$ ' or ' $\mathrm{NO}_{\mathrm{x}}$ ' or ' $\mathrm{SO}_{2}$ ' or 'pollution'. The inclusion criteria included 
i) peer-reviewed publications reporting original data; ii) a minimum of 10 adult subjects tested to maximize reliability; and iii) arterial stiffness measured with a well-accepted and validated technique [pulse wave velocity (PWV)]; reflected waves estimated by augmentation index or augmentation pressure. Two independent reviewers (L.Z. and P.L.) selected the studies for inclusion in this systematic review. First, the titles of the studies were screened for relevance. In case of doubt, conflict or discussion between the two independent reviewers, the article was retained. Second, publications with titles or abstracts appearing to meet these inclusion criteria were selected for detailed review. In cases of doubt on the inclusion of an article, a decision was achieved by consensus. The reference lists of the analysed studies were also searched. These studies were subjected to the same selection procedures. A narrative of the collected data has been reported. Because of the high heterogeneity of the studies, a meta-analysis was not performed. Whenever possible, data were presented as the mean \pm standard deviation and percentages.

\section{Results}

The PRISMA flow diagram is reported in Fig. 1, summarizing the number of studies included for analysis based on the search criteria of this systematic review. Using the search terms, a total of 76 studies were identified. In total, 52 studies were excluded based on a review of the title and the abstract only; 16 studies were excluded after the reading of the full text. The remaining 8 studies, published within the last 8 years, reported carotid-femoral PWV (2 studies), brachial-ankle PWV (2 studies), augmentation index and/or augmentation pressure measurements (6 studies) and were considered for the review (19-26). The main results of the studies included in this review are reported in Table I.

Exposure to air pollutants and arterial stiffness. The exposure to particulate matter pollutants $\left(\mathrm{PM}_{2.5}, \mathrm{PM}_{10}\right)$ was evaluated in 8 studies (19-26). In 6 of them (19,22-26), the exposure was associated with an increased arterial stiffness and/or wave reflection (estimated by brachial-ankle PWV, augmentation index and augmentation pressure) whereas in the remaining two studies $(20,21)$ the exposure was not associated with either carotid-femoral PWV or augmentation index. However, in one of these negative studies (20), the $\mathrm{PM}_{2.5}$ exposure variability within the urban population was very low (mean $\mathrm{PM}_{2.5}$ exposure: $21.4 \pm 1.1 \mu \mathrm{g} / \mathrm{m}^{3}$ ); in the second negative study (21), despite the augmentation index was slightly increased $(25.4 \pm 4.8 \%$ vs. $22.6 \pm 5.1 \%$ ) in the group of patients with the higher $\mathrm{PM}_{2.5}$ exposure, this difference was not significant, probably for the small sample size $(n=23)$. The exposure to gaseous pollutants $\left(\mathrm{NO}_{2}, \mathrm{NO}_{\mathrm{x}}, \mathrm{SO}_{2}\right)$ was evaluated in 3 studies $(20,22,24)$ and was associated with increased arterial stiffness and wave reflection in two large studies $(20,22)$ whereas no significant association with brachial-ankle PWV was observed for $\mathrm{NO}_{2}$ in the third, smaller study (24).

Increased arterial stiffness and wave reflection were reported in 4 of the 5 studies that evaluated the short-term exposure to particulate matter pollutants $(19,21,22,24,25)$ and in 2 of the 3 studies that evaluated the long-term exposure to particulate matter pollutants $(20,23,26)$, whereas an increased

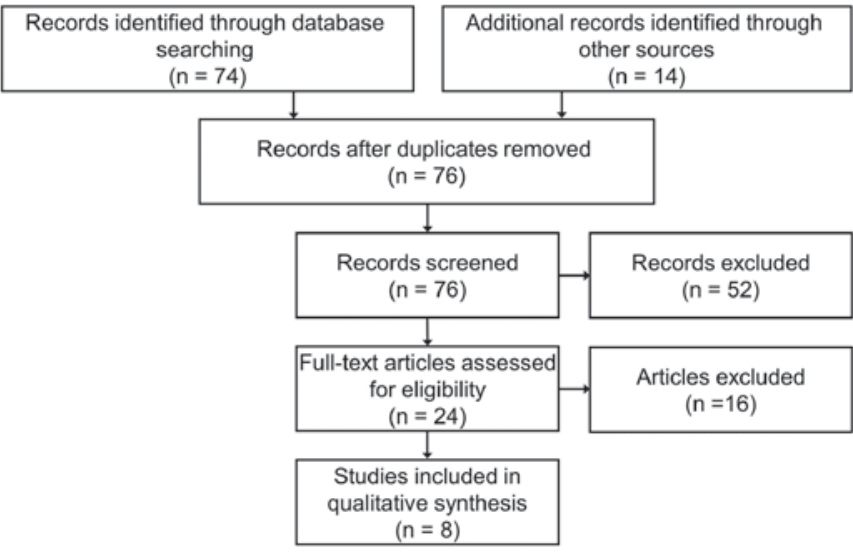

Figure 1. PRISMA flow diagram.

wave reflection was reported in 1 of the 2 studies that evaluated the short-term ( $\leq 1$ day) exposure to gaseous pollutants $(22,24)$, whereas both increased arterial stiffness and wave reflection were reported in one article after long-term exposure to gaseous pollutants (20).

The association between serum biomarkers of vascular inflammation and air pollutants was evaluated in 3 studies $(21,24)$. One study $(24)$ evaluated the effect of $\mathrm{NO}_{2}$ exposure on hsCRP and revealed a positive association between 1-day $\mathrm{NO}_{2}$ exposure and hsCRP levels. No significant association was observed between $\mathrm{PM}_{2.5}$ levels and hsCRP in either study $(21,24)$. The association between serum biomarkers of inflammation and arterial stiffness was evaluated only in one study (26) in which hsCRP was positively correlated with brachial-ankle PWV.

\section{Discussion}

In the present systematic review of the effect of air pollution on arterial stiffness and wave reflection, we included 8 studies for discussion. A total of 6 of the 8 studies reported an increased arterial stiffness and/or wave reflection after particulate matter pollutants exposure. The exposure to gaseous pollutants was associated with increased arterial stiffness and wave reflection in 2 of the 3 studies with available data.

Increased arterial stiffness and wave reflection after exposure to air pollutants. Possible underlying mechanisms. During the last decades, several clinical settings, either physiological or pathological, has been associated with increased arterial stiffness and wave reflection. Most of them, such as the elderly and chronic diseases, are irreversible, other clinical conditions, such as chronic inflammation, are associated with increased arterial stiffness $(4-12,27)$ and potentially reversible, as suggested in recent studies showing that the reduction of inflammation can be associated with a reduction of arterial stiffness $(6,8,27)$. In this review, we reported that arterial stiffness is increased after exposure of air pollutants. Despite it remains to be demonstrated whether the reduction of chronic exposure to air pollution is associated with arterial destiffening, it was reported that wave reflection increased immediately after $1 \mathrm{~h}$ of exposure to diesel exhaust while there was no effect on arterial stiffness $40 \mathrm{~min}$ after exposure (28), 


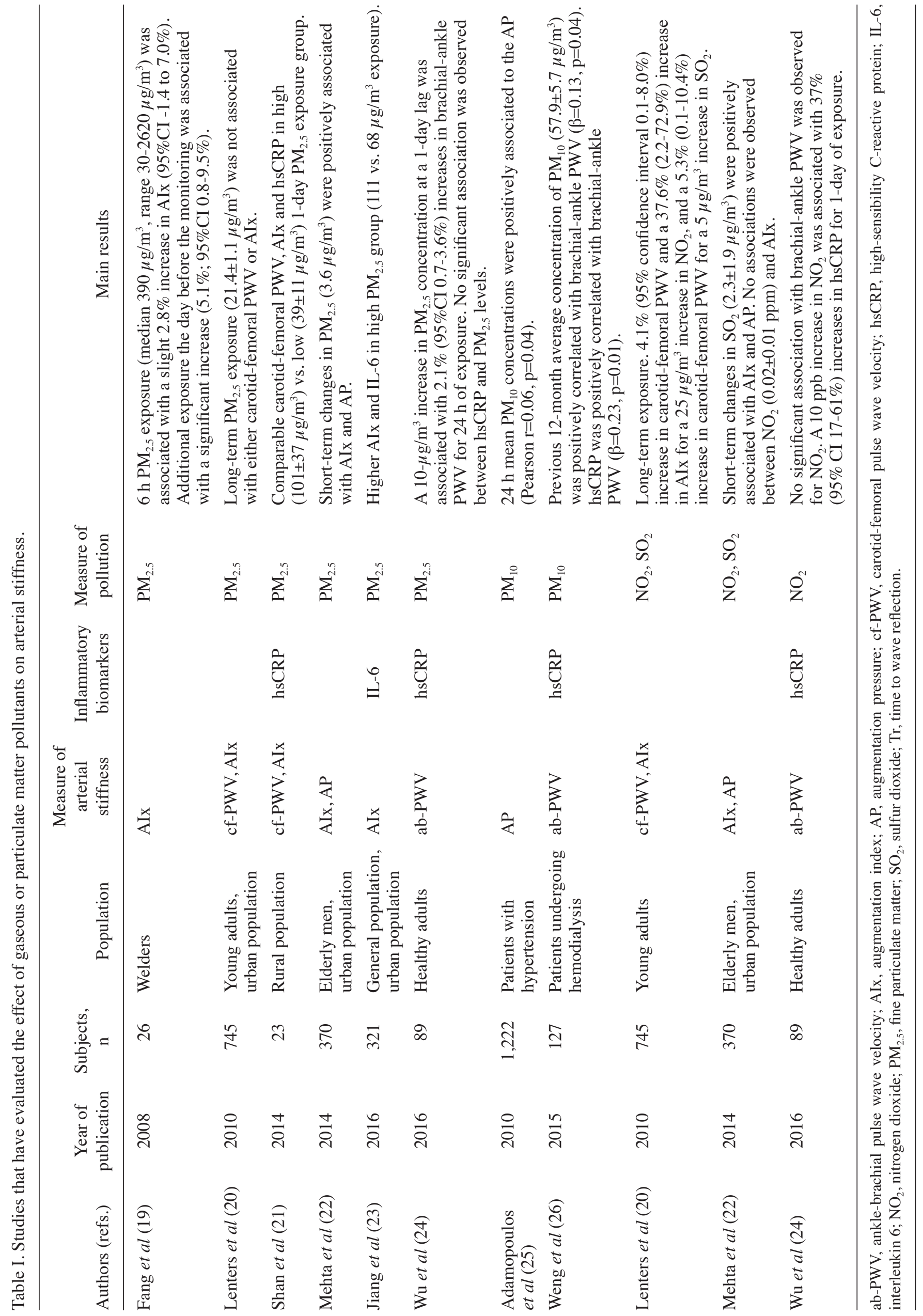


suggesting that acute exposure to pollutants could be associated with reversible (functional) arterial stiffening.

Several mechanisms involved in the stiffening of large arteries after exposure to air pollution could be involved. In this regard, acute and chronic inflammation could play a role in this process. Despite only one of the studies included in this review explored the association between air pollution and a biomarker of vascular inflammation (24), the negative effects of air pollution on inflammatory state are well known and widely documented in literature.

In another model of chronic inflammation it was reported that inflammation may be associated with functional or structural arterial stiffening (12). Functional arterial stiffening could be the consequence of endothelial dysfunction, reduced production/increased inactivation of nitric oxide and reduced vasodilation whereas structural arterial stiffening can be associated with the production of uncoiled stiff collagen, degradation of elastin, smooth muscle cell migration and intima proliferation, vascular calcification and stiffening of the extracellular matrix. The increased arterial stiffness after short-term exposure to air pollutants is in agreement with the finding that even an acute, mild and transitory inflammatory stimulus is associated with arterial stiffening (29) and suggests a functional arterial stiffening. In support of this hypothesis, there is evidence that particulate matter pollutants exposure is associated with acute arterial vasoconstriction (16) and endothelial dysfunction (14,16-18). Moreover, the increase in augmentation index and augmentation pressure, two indices of increased backward reflected waves, after exposure to air pollution $(19,22,23,25)$ may be the consequence of the vasoconstriction at the level of the microcirculation (25). Whether the long-term exposure to air pollutants is associated with functional or structural arterial stiffening is not known because none of the studies included in this review explored the effect of the reduction of long-term exposure to air pollution.

In presence of increased arterial stiffness, the destiffening of large arteries is an objective with important implications on the cardiovascular risk, as suggested by the increased survival after the reduction of arterial stiffness (30). To obtain an arterial destiffening, three levels of treatment can be used. The first is the prescription of a drug that reduces the arterial stiffness, such as an antihypertensive with an independent effect on arterial stiffness. Unfortunately, using this option the causal factors of the arterial stiffening remain active. The second level of treatment consists in the reduction, most probably, or elimination, hardly, of a known causal factor of arterial stiffening, i.e. the chronic inflammation $(6,8,27)$. This approach, acting in a step before the arterial wall stiffening, can be potentially highly effective in single patients. Regarding this point, interventional multicentre studies are ongoing. The model studied in this review has a great clinical importance because, reducing the air pollution, we can reduce the arterial stiffening already occurred and also prevent the development of chronic inflammation, a causal factor of arterial stiffening and several cardiovascular complications, acting at a population-based level of treatment. Consequently, the evidence that air pollution is associated with increased arterial stiffness has also a potential great epidemiological relevance and could represent a warning for the lawmakers and the occupational physicians.
Methodological issues. The present study has several strengths. First, to the best of our knowledge, no systematic review studies have determined whether arterial stiffness is increased after air pollution. Second, we used widely accepted measures of arterial stiffness and wave reflection. PWV represents the speed at which the pressure wave is generated by left-ventricular contraction is transmitted within the arterial tree. The carotid-femoral PWV represents the gold standard for arterial stiffness assessments in daily practice whereas ankle-brachial PWV represents the stiffness of muscular and elastic arteries considered as a whole. Augmentation index and augmentation pressure are measures of wave reflection. This study may have also a number of potential limitations. First, all of the studies included in this review did not explore the effect of the reduction of exposure to air pollutants on arterial stiffness and reflection waves. Future prospective studies need to be performed to confirm the hypothesis of a destiffening effect of a population-based reduction of the air pollution. Second, due to the high heterogeneity of the time of exposure and the concentration of air pollutants, outcomes and population studied included in this systematic review, a meta-analysis was not performed. Third, we con not exclude that the presence of comorbidities could have influenced the results of the studies included in this review. Adamopoulos et al (25) included patients with hypertension, Weng et al included patients undergoing haemodialysis (26), whereas other authors (19-23) included patients from the general population, and did not report any exclusion criteria or included patients with several cardiovascular risk factors.

In conclusion, available evidence supports an association of gaseous and particulate matter pollutants with an increased arterial stiffness and wave reflection. These findings may have important clinical implications for population-based strategies aimed at the reduction of arterial stiffness, an intermediate end-point of cardiovascular diseases.

\section{References}

1. Vlachopoulos C, Xaplanteris P, Aboyans V, Brodmann M, Cífková R, Cosentino F, De Carlo M, Gallino A, Landmesser U, Laurent $\mathrm{S}$, et al: The role of vascular biomarkers for primary and secondary prevention. A position paper from the European Society of Cardiology Working Group on peripheral circulation: Endorsed by the Association for Research into Arterial Structure and Physiology (ARTERY) Society. Atherosclerosis 241: 507-532, 2015.

2. Heo J, Schauer JJ, Yi O, Paek D, Kim H and Yi SM: Fine particle air pollution and mortality: Importance of specific sources and chemical species. Epidemiology 25: 379-388, 2014.

3. Pope CA III, Burnett RT, Thurston GD, Thun MJ, Calle EE, Krewski D and Godleski JJ: Cardiovascular mortality and long-term exposure to particulate air pollution: Epidemiological evidence of general pathophysiological pathways of disease. Circulation 109: 71-77, 2004.

4. Zanoli L, Inserra G and Castellino P: Increased cardiovascular risk in subjects with a low prevalence of classic cardiovascular risk factors: The inflammatory bowel disease paradox. Trends Cardiovasc Med 25: 705-706, 2015.

5. Zanoli L, Cannavò M, Rastelli S, Di Pino L, Monte I, Di Gangi M, Boutouyrie P, Inserra G, Laurent S and Castellino P: Arterial stiffness is increased in patients with inflammatory bowel disease. J Hypertens 30: 1775-1781, 2012.

6. Zanoli L, Rastelli S, Granata A, Inserra G, Empana JP, Boutouyrie P, Laurent S and Castellino P: Arterial stiffness in inflammatory bowel disease: A systematic review and meta-analysis. J Hypertens 34: 822-829, 2016. 
7. Zanoli L, Granata A, Lentini P, Gaudio A and Castellino P Augmentation index is increased in patients with inflammatory bowel disease, a meta-analysis. Eur J Intern Med: Dec 21, 2016 (Epub ahead of print). https://doi.org/10.1016/j.ejim.2016.12.012.

8. Zanoli L, Rastelli S, Inserra G, Lentini P, Valvo E, Calcagno E, Boutouyrie P, Laurent S and Castellino P: Increased arterial stiffness in inflammatory bowel diseases is dependent upon inflammation and reduced by immunomodulatory drugs. Atherosclerosis 234: 346-351, 2014

9. Zanoli L, Boutouyrie P, Lentini P, Rastelli S and Castellino P: Maintenance therapy with salicylates is associated with aortic stiffening in patients withinflammatory boweldisease.JHypertens (In press). https://doi.org/10.1097/HJH.0000000000001235.

10. Zanoli L, Signorelli SS, Inserra G and Castellino P: Subclinical atherosclerosis in patients with inflammatory bowel diseases: A systematic review and meta-analysis. Angiology: Oct 26, 2016 (Epub ahead of print). doi: 10.1177/ 0003319716675076.

11. Wu GC, Leng RX, Lu Q, Fan YG, Wang DG and Ye DQ: Subclinical atherosclerosis in patients with inflammatory bowel diseases: A systematic review and meta-analysis. Angiology: Jun 1, 2016 (Epub ahead of print).

12. Zanoli L, Rastelli S, Inserra G and Castellino P: Arterial structure and function in inflammatory bowel disease. World $J$ Gastroenterol 21: 11304-11311, 2015.

13. Peters A, Fröhlich M, Döring A, Immervoll T, Wichmann HE, Hutchinson WL, Pepys MB and Koenig W: Particulate air pollution is associated with an acute phase response in men results from the MONICA-Augsburg Study. Eur Heart J 22: 1198-1204, 2001.

14. Tamagawa E, Bai N, Morimoto K, Gray C, Mui T, Yatera K, Zhang X, Xing L, Li Y, Laher I, et al: Particulate matter exposure induces persistent lung inflammation and endothelial dysfunction. Am J Physiol Lung Cell Mol Physiol 295: L79-L85, 2008.

15. Briet M, Collin C, Laurent S, Tan A, Azizi M, Agharazii M, Jeunemaitre X, Alhenc-Gelas F and Boutouyrie P: Endothelial function and chronic exposure to air pollution in normal male subjects. Hypertension 50: 970-976, 2007.

16. Brook RD, Brook JR, Urch B, Vincent R, Rajagopalan S and Silverman F: Inhalation of fine particulate air pollution and ozone causes acute arterial vasoconstriction in healthy adults. Circulation 105: 1534-1536, 2002.

17. Urch B, Silverman F, Corey P, Brook JR, Lukic KZ, Rajagopalan S and Brook RD: Acute blood pressure responses in healthy adults during controlled air pollution exposures. Environ Health Perspect 113: 1052-1055, 2005.

18. Rundell KW, Hoffman JR, Caviston R, Bulbulian R and Hollenbach AM: Inhalation of ultrafine and fine particulate matter disrupts systemic vascular function. Inhal Toxicol 19: 133-140, 2007.

19. Fang SC, Eisen EA, Cavallari JM, Mittleman MA and Christiani DC: Acute changes in vascular function among welders exposed to metal-rich particulate matter. Epidemiology 19: 217-225, 2008.
20. Lenters V, Uiterwaal CS, Beelen R, Bots ML, Fischer P, Brunekreef B and Hoek G: Long-term exposure to air pollution and vascular damage in young adults. Epidemiology 21: 512-520, 2010.

21. Shan M, Yang X, Ezzati M, Chaturvedi N, Coady E, Hughes A, Shi Y, Yang M, Zhang Y and Baumgartner J: A feasibility study of the association of exposure to biomass smoke with vascular function, inflammation, and cellular aging. Environ Res 135: 165-172, 2014.

22. Mehta AJ, Zanobetti A, Koutrakis P, Mittleman MA, Sparrow D, Vokonas P and Schwartz J: Associations between short-term changes in air pollution and correlates of arterial stiffness: The Veterans Affairs Normative Aging Study, 2007-2011. Am J Epidemiol 179: 192-199, 2014.

23. Jiang S, Bo L, Gong C, Du X, Kan H, Xie Y, Song W and Zhao J: Traffic-related air pollution is associated with cardio-metabolic biomarkers in general residents. Int Arch Occup Environ Health 89: 911-921, 2016.

24. Wu CF, Shen FH, Li YR, Tsao TM, Tsai MJ, Chen CC, Hwang JS, Hsu SH, Chao H, Chuang KJ, et al: Association of short-term exposure to fine particulate matter and nitrogen dioxide with acute cardiovascular effects. Sci Total Environ 569-570: 300-305, 2016.

25. Adamopoulos D, Vyssoulis G, Karpanou E, Kyvelou SM, Argacha JF, Cokkinos D, Stefanadis C and van de Borne P: Environmental determinants of blood pressure, arterial stiffness, and central hemodynamics. J Hypertens 28: 903-909, 2010.

26. Weng CH, Hu CC, Yen TH and Huang WH: Association between environmental particulate matter and arterial stiffness in patients undergoing hemodialysis. BMC Cardiovasc Disord 15: 115, 2015.

27. Mäki-Petäjä KM, Hall FC, Booth AD, Wallace SM, Yasmin, Bearcroft PW, Harish S, Furlong A, McEniery CM, Brown J, et al: Rheumatoid arthritis is associated with increased aortic pulse-wave velocity, which is reduced by anti-tumor necrosis factor-alpha therapy. Circulation 114: 1185-1192, 2006.

28. Lundbäck M, Mills NL, Lucking A, Barath S, Donaldson K, Newby DE, Sandström T and Blomberg A: Experimental exposure to diesel exhaust increases arterial stiffness in man. Part Fibre Toxicol 6: 7, 2009.

29. Vlachopoulos C, Dima I, Aznaouridis K, Vasiliadou C, Ioakeimidis N, Aggeli C, Toutouza M and Stefanadis C: Acute systemic inflammation increases arterial stiffness and decreases wave reflections in healthy individuals. Circulation 112: 2193-2200, 2005.

30. Guerin AP, Blacher J, Pannier B, Marchais SJ, Safar ME and London GM: Impact of aortic stiffness attenuation on survival of patients in end-stage renal failure. Circulation 103: 987-992, 2001. 